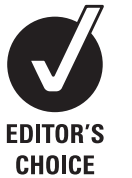

1 Department of Cardiology, Concord Repatriation General Hospital, University of Sydney, Concord, New South Wales, Australia

${ }^{2}$ Department of Biochemistry, Concord Repatriation General Hospital, University of Sydney, Concord, New South Wales, Australia

\section{Correspondence to}

Professor David B Brieger Department of Cardiology. Concord Repatriation General Hospital, Hospital Road, Concord, NSW 2139, Australia; briegster@gmail.com

Relationship with industry: The high-sensitivity troponin T assays were provided by Roche Diagnostics.

Accepted 4 March 2012

ORIGINAL ARTICLE

\title{
Perioperative myocardial necrosis in patients at high cardiovascular risk undergoing elective non-cardiac surgery
}

\author{
Richard F Alcock, ${ }^{1}$ Dorothy Kouzios, ${ }^{2}$ Christopher Naoum, ${ }^{1}$ Graham S Hillis, ${ }^{1}$ \\ David B Brieger ${ }^{1}$
}

\begin{abstract}
Objective Cardiovascular complications are important causes of morbidity and mortality in elective non-cardiac surgery. Although difficult to diagnose, perioperative myocardial infarction (MI) remains prognostically important. High-sensitivity troponin T (hs-TnT) assays allow detection of very minor damage to cardiac muscle. These assays are yet to be fully evaluated in the perioperative setting. Our aim was to determine the incidence and predictors of myocardial necrosis in patients at high cardiovascular risk undergoing elective non-cardiac surgery using hs-TnT.

Design Prospective observational cohort study. Patients 352 consecutive patients undergoing elective major non-cardiac surgery prescribed antiplatelet therapy for primary or secondary cardiovascular event prevention. Main outcome measure The incidence of elevated preoperative hs-TnT ( $\geq 14 \mathrm{ng} /$ litre), hs-TnT-defined perioperative myocardial necrosis ( $\geq 14 \mathrm{ng} /$ litre and $50 \%$ increase from preoperative level), and perioperative $\mathrm{Ml}$ were determined in relation to patient and surgical factors.
\end{abstract}

Results Preoperative hs-TnT was elevated in $31 \%$ and postoperative myocardial necrosis occurred in $22 \%$ of patients. Predictors of elevated baseline hs-TnT included age (OR 1.10, $\mathrm{p}<0.001$ ), male gender (OR 2.91, $p<0.001$ ), diabetes requiring insulin therapy (OR 4.85 , $p=0.004)$ and chronic kidney disease (OR 3.60, $p<0.001)$. Independent predictors of perioperative myocardial necrosis were age (OR 1.07, $p<0.001)$, intraoperative hypotension (OR 3.67, $p=0.001$ ) and orthopaedic surgery (OR 2.46, $p=0.005$ ). Only $2 \%$ of patients suffered clinically apparent MI. Elevated preoperative hs-TnT did not predict perioperative myocardial necrosis or Ml.

Conclusions Perioperative myocardial damage occurs frequently in patients undergoing elective non-cardiac surgery, although the majority of events are clinically undetected. Age and intraoperative hypotension are independent predictors of myocardial necrosis in this setting.

\section{INTRODUCTION}

The incidence and prevalence of cardiovascular disease increases with age and it is expected that both will continue to rise with the ageing population. Similarly, the majority of surgical procedures are undertaken in older patients, with an estimated 200 million major operations performed annually worldwide. ${ }^{1}$ Although such surgery has obvious benefits there are associated risks. In particular, cardiovascular complications are important causes of morbidity and mortality in patients undergoing elective non-cardiac surgery, with adverse cardiac outcomes estimated to occur in approximately $4 \%$ of all patients. ${ }^{2-4}$ The risk among those with established ischaemic heart disease or at increased cardiovascular risk is even higher, with the incidence of perioperative myocardial infarction (MI) ranging from $4 \%$ to $16 \%,{ }^{5}$ depending on the precise characteristics of the population and the definitions used.

Although MI may complicate elective noncardiac surgery, early detection remains a challenge in the perioperative setting. Myocardial ischaemia and MI frequently occur without symptoms or ECG changes. ${ }^{7}$ Even in the absence of clinically apparent symptoms, perioperative MI remains prognostically important. $^{8}$

Cardiac troponins are highly sensitive and specific biochemical markers for myocardial necrosis and are fundamental to the diagnosis of acute $\mathrm{MI},{ }^{9}$ in addition to predicting longer-term morbidity and mortality. ${ }^{10}$ Similarly, cardiac troponin elevation in the postoperative setting has been shown to predict adverse short-term and longer-term outcomes. ${ }^{11} 12$ The development of high-sensitivity assays has greatly improved the analytical performance of cardiac troponin, allowing detection of very minor damage to cardiac muscle. ${ }^{13}$ In particular, elevated levels of highsensitivity troponin $\mathrm{T}$ (hs- $\mathrm{Tn} \mathrm{T}$ ) are detectable in stable populations, with prognostic significance. ${ }^{14}$

The aims of our study were to identify the incidence of elevated preoperative hs-TnT in higherrisk patients undergoing non-cardiac surgery, to determine the incidence and predictors of perioperative myocardial necrosis, and to investigate the association between elevated preoperative hsTnT and perioperative myocardial necrosis and infarction.

\section{METHODS \\ Patient selection}

Consecutive patients presenting to a tertiary referral centre for preoperative anaesthetic evaluation between January and November 2011 were recruited. Patients aged 45 years or older, undergoing major elective non-cardiac surgery (defined by procedures requiring a hospital admission of $24 \mathrm{~h}$ or 
longer), and receiving antiplatelet therapy for primary or secondary cardiovascular event prevention were eligible for inclusion in the study. Detailed clinical evaluation by an experienced anaesthetist and laboratory testing were performed preoperatively in all patients. The institutional Human Research and Ethics Committee approved the study protocol and written informed consent was obtained from all participants.

Perioperative cardiac risk was estimated using the Revised Cardiac Risk Index. ${ }^{2}$ This includes six equally weighted clinical variables: history of ischaemic heart disease; history of congestive cardiac failure; cerebrovascular disease; diabetes requiring insulin therapy; impaired renal function (serum creatinine $>176 \mu \mathrm{mol} /$ litre); and high-risk surgery (intraperitoneal, intrathoracic or suprainguinal vascular procedures). Moderate risk is defined as two risk factors and high risk as three or more risk factors, corresponding to a perioperative risk for an adverse cardiac event of $7 \%$ and $11 \%$ respectively. ${ }^{2}$

\section{Perioperative antiplatelet therapy management}

Management of antiplatelet therapy in the perioperative period was left to the discretion of the anaesthetist performing the preoperative evaluation and the treating surgeon. Cardiology involvement in decision-making was also at the discretion of the assessing anaesthetist. Continuation or discontinuation of antiplatelet therapy, as well as the time interval between therapy cessation and surgical procedure were recorded. Patients on dual antiplatelet therapy (ie, aspirin and clopidogrel) were categorised as having antiplatelet therapy ceased only if both agents were discontinued.

\section{Cardiac troponin $\mathbf{T}$ and myocardial events}

Baseline preoperative hs-TnT (Roche Diagnostics, Mannheim, Germany; 99th percentile $13 \mathrm{ng} /$ litre, lower limit of detection $5 \mathrm{ng} /$ litre, assay linearity to $10000 \mathrm{ng} /$ litre) ${ }^{15}$ were collected from patients at preoperative assessment in the week preceding scheduled surgery, with postoperative samples collected at 24 and $48 \mathrm{~h}$ following surgery. Fourth-generation cardiac troponin $\mathrm{T}$ (Roche Diagnostics; 99th percentile $10 \mathrm{ng} / \mathrm{litre}$ ) was also determined in the initial 86 patients recruited. A baseline elevation of hs-TnT was defined as a concentration above the 99th percentile reference range for the assay ( $\geq 14 \mathrm{ng} /$ litre). Perioperative myocardial necrosis was defined as an hs-TnT concentration above the 99th percentile ( $\geq 14 \mathrm{ng} /$ litre) and a delta value of $50 \% .{ }^{16}$ Further analysis was also performed using a comparison delta value of $30 \%$. Clinically apparent perioperative MI was defined using the current Universal Definition for $\mathrm{MI},{ }^{17}$ which outside the setting of myocardial revascularisation requires the presence of typical ischaemic symptoms, ECG changes indicative of ischaemia or new pathological Q-waves, or subsequent imaging evidence of MI, in conjunction with myocardial necrosis

\section{Intraoperative variables}

The method of anaesthesia, intraoperative haemodynamics and length of surgery were collected. There were no protocol-based restrictions of anaesthetic or surgical technique, and the primary anaesthesia providers made all clinical decisions as per their routine practice. Haemodynamic variables (blood pressure and heart rate) were electronically recorded at 5-min intervals throughout the procedure. Abnormal intraoperative haemodynamics were defined as hypotension (systolic BP $<100 \mathrm{~mm} \mathrm{Hg}$ ), hypertension (systolic BP >160 mm Hg), bradycardia (heart rate $<55$ beats per minute) and tachycardia (heart rate $>100$ beats per minute), with two or more sequential readings at least 5 min apart defining occurrence.

\section{Adverse perioperative cardiovascular outcomes}

Patients and medical records were reviewed daily until hospital discharge. Study events comprised adverse cardiovascular outcomes during the index hospitalization, including major cardiac events (death and clinically apparent MI), myocardial necrosis (hs-TnT concentration $\geq 14 \mathrm{ng} /$ litre and a delta value of $50 \%$ ), cerebrovascular events (defined as a new focal neurological deficit thought to be vascular in origin with signs and symptoms lasting more than $24 \mathrm{~h}$ ), atrial fibrillation (defined as new atrial fibrillation resulting in angina, congestive heart failure, symptomatic hypotension or requiring pharmacological treatment, or electrical cardioversion) and venous thromboembolic disease (deep venous thrombosis or pulmonary embolism). Perioperative bleeding complications were classified according to the Thrombolysis in Myocardial Infarction definition: major bleed as intracranial haemorrhage or a $>5 \mathrm{~g} / \mathrm{dl}$ decrease in haemoglobin concentration, minor bleed as a $3 \mathrm{~g} / \mathrm{dl}$ to $\leq 5 \mathrm{~g} / \mathrm{dl}$ decrease in haemoglobin concentration. In addition the requirement of blood transfusion, as determined by the treating surgical team, was recorded.

\section{Statistical analysis}

Continuous data are expressed as mean (SD) and median (IOR) and categorical data as total number of patients (percentages). Predictors of both baseline elevated hs-TnT and perioperative myocardial necrosis were identified using binary logistic regression and the relationship expressed as an OR (95\% CI). Multivariable analysis was performed using stepwise binary logistic regression. All variables predictive of perioperative myocardial necrosis with a $\mathrm{p}$ value $<0.10$ on univariable analysis were included in the multivariable analysis. A two-tailed $p$ value $<0.05$ was considered significant. All the analyses were performed using SPSS V.15.0 (SPSS Inc).

\section{RESULTS}

The study cohort consisted of 352 consecutive patients undergoing major elective non-cardiac surgery, prescribed antiplatelet therapy for primary or secondary cardiovascular prevention. The mean (SD) patient age was 72 (10) years and 64\% were men (table 1). The relatively high prevalence of cardiovascular risk factors, comorbidities and medications reflect our patient selection criteria. The listed baseline medications reflect chronic prescription at the time of preoperative assessment. Although all patients either had established cardiovascular disease or were at high risk of the disease, the Revised Cardiac Risk Index suggested that the majority of our cohort were at low risk for a major adverse perioperative cardiovascular outcome (table 1).

\section{Baseline cardiac hs-TnT}

Preoperative baseline hs-TnT was elevated above the reference 99th percentile in $31 \%$ of patients, with the majority of elevated levels falling just above the $14 \mathrm{ng} /$ litre threshold (figure 1 ). The independent predictors of an elevated baseline hs-TnT included age (HR 1.10; 95\% CI 1.06 to 1.14, $\mathrm{p}<0.001)$, male gender (HR 2.91; $95 \%$ CI 1.61 to 5.26, $\mathrm{p}<0.001)$, diabetes requiring insulin therapy (HR 4.85; 95\% CI 1.85 to $12.72, \mathrm{p}=0.004$ ) and chronic kidney disease (HR 3.60; 95\% CI 2.09 to 6.19, p<0.001) defined as an estimated glomerular filtration rate $<60 \mathrm{ml} / \mathrm{min}$ per $1.73 \mathrm{~m}^{2}$. 
Table 1 Baseline characteristics

\begin{tabular}{|c|c|}
\hline Variable & Study population \\
\hline Age, years $\pm S D$ & $72.2 \pm 9.6$ \\
\hline Median (25-75th percentile) & $73(65-79)$ \\
\hline Male gender, n (\%) & $226(64)$ \\
\hline \multicolumn{2}{|l|}{ Comorbid conditions, n (\%) } \\
\hline Ischaemic heart disease & $152(43)$ \\
\hline Congestive cardiac failure & $10(3)$ \\
\hline Atrial fibrillation (permanent or paroxysmal) & $34(10)$ \\
\hline Previous coronary stent & $53(15)$ \\
\hline Previous coronary bypass surgery & $70(20)$ \\
\hline Cerebrovascular disease & $53(15)$ \\
\hline Peripheral vascular disease & $60(17)$ \\
\hline Hypertension & $274(78)$ \\
\hline Diabetes mellitus & $121(34)$ \\
\hline Diabetes mellitus requiring insulin & $27(7)$ \\
\hline Current smoker & $28(8)$ \\
\hline Ex smoker & $139(39)$ \\
\hline Obstructive sleep apnoea & $21(6)$ \\
\hline Renal impairment (eGFR $<60 \mathrm{ml} / \mathrm{min}$ per $1.73 \mathrm{~m}^{2}$ ) & $106(30)$ \\
\hline \multicolumn{2}{|l|}{ Medications, n (\%) } \\
\hline Aspirin & $300(85)$ \\
\hline Clopidogrel & $77(22)$ \\
\hline Dipyridamole and aspirin combination & $10(3)$ \\
\hline Prasugrel & $1(<1)$ \\
\hline Dual antiplatelet therapy & $36(10)$ \\
\hline ACE-I/ARB & $243(68)$ \\
\hline Statin & $239(68)$ \\
\hline$\beta$ blocker & $114(32)$ \\
\hline Calcium channel blocker & $97(28)$ \\
\hline Digoxin & $8(2)$ \\
\hline Nitrate & $36(10)$ \\
\hline \multicolumn{2}{|l|}{ Revised cardiac risk index, $\mathrm{n}(\%)$} \\
\hline 0 & $133(38)$ \\
\hline 1 & $146(41)$ \\
\hline 2 & $57(16)$ \\
\hline$\geq 3$ & $16(5)$ \\
\hline \multicolumn{2}{|l|}{ Baseline hs-TnT (ng/litre) } \\
\hline Mean $\pm S D$ & $15.7 \pm 27.4$ \\
\hline Median (25-75th percentile) & $9.0(4.0-16.9)$ \\
\hline Elevated $\geq 14$ ng/litre, n (\%) & $109(31)$ \\
\hline
\end{tabular}

Values are expressed as mean $\pm \mathrm{SD}, \mathrm{n}(\%)$ or median (IOR).

ACE-I, ACE inhibitor; ARB, angiotensin II receptor blocker; eGFR, estimated glomerular filtration rate; hs-TnT, high-sensitivity troponin $\mathrm{T}$.

\section{Surgical and procedural variables}

All major non-cardiac surgical procedures were represented in our study with the exception of major thoracic surgery, which is not routinely performed at our institution. General anaesthesia was the preferred anaesthetic technique in the majority of cases, either alone or in combination with other methods. Intraoperative hypotension was the predominant haemodynamic disturbance, occurring in $70 \%$ of patients, with mean hypotensive duration of $33 \mathrm{~min}$. The wide SD in duration of surgery and postoperative length of stay reflects the heterogeneity of surgical procedures included (table 2). Compared with other methods of anaesthesia, only general anaesthesia was significantly associated with intraoperative hypotension (OR 4.58; 95\% CI 2.53 to 8.30, $\mathrm{p}<0.001)$

\section{Perioperative antiplatelet therapy management}

Antiplatelet therapy was prescribed for secondary cardiovascular prevention in 235 (67\%) of patients, including 152 (43\%) for ischaemic heart disease, with $53(15 \%)$ having previous coronary artery stenting and $70(20 \%)$ having previously undergone

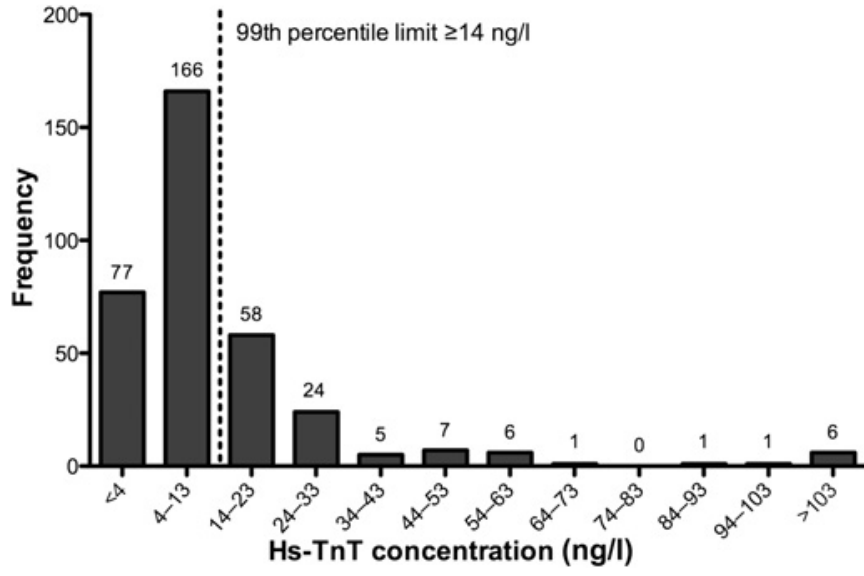

Figure 1 Distribution of baseline high-sensitivity troponin $\mathrm{T}(\mathrm{TnT})$ concentration. The dashed line represents the 99 th percentile ( $\geq 14 \mathrm{ng} /$ litre) reference concentration from a healthy population.

coronary artery bypass grafting. All antiplatelet therapy was ceased in $280(80 \%)$ of all patients in the perioperative period, including $70 \%$ of patients with ischaemic heart disease and $50 \%$ with previous coronary stenting. With the exception of major vascular procedures, antiplatelet therapy was ceased in the majority of patients (table 3 ). The management of antiplatelet therapy had no impact on the occurrence of perioperative minor or major bleeding complications, or the need for blood transfusion.

\section{Adverse perioperative cardiovascular events}

Myocardial necrosis occurred in $22 \%$ of patients undergoing an elective major non-cardiac surgical procedure (table 4). Myocardial necrosis, utilising the alternative $30 \%$ delta

Table 2 Surgical and procedural variables

\begin{tabular}{|c|c|}
\hline Variable & Study population \\
\hline \multicolumn{2}{|l|}{ Surgical procedure, $\mathrm{n}(\%)$} \\
\hline Major vascular & $52(15)$ \\
\hline Major orthopaedic & $80(23)$ \\
\hline General & $48(13)$ \\
\hline Major urological & $66(19)$ \\
\hline Major neurosurgery & $18(5)$ \\
\hline Lower risk & $88(25)$ \\
\hline \multicolumn{2}{|l|}{ Anaesthesia, n (\%) } \\
\hline General & $294(84)$ \\
\hline Spinal & $64(18)$ \\
\hline Epidural & $5(1)$ \\
\hline Nerve block & $53(15)$ \\
\hline \multicolumn{2}{|l|}{ Intraoperative haemodynamics, n (\%) } \\
\hline Hypotension (SBP $<100 \mathrm{~mm} \mathrm{Hg}$ ) & $245(70)$ \\
\hline Duration of hypotension (mean $\pm S D$, min) & $33 \pm 24$ \\
\hline Hypertension (SBP >160 mm Hg) & $123(35)$ \\
\hline Bradycardia (HR $<55$ bpm) & $67(19)$ \\
\hline Tachycardia (HR >100 bpm) & $15(4)$ \\
\hline \multicolumn{2}{|l|}{ Duration of surgery } \\
\hline Mean $\pm S D, \min$ & $117 \pm 73$ \\
\hline Median, min (25-75th percentile) & $105(67-147)$ \\
\hline \multicolumn{2}{|l|}{ Length of stay, days } \\
\hline Mean $\pm S D$ & $5.6 \pm 6.7$ \\
\hline Median (25-75th percentile) & $4(2-7)$ \\
\hline
\end{tabular}


Table 3 Perioperative management of antiplatelet therapy

\begin{tabular}{lccc}
\hline Variable & Number of patients & Antiplatelet therapy continued & Antiplatelet therapy ceased \\
\hline All patients, $\mathrm{n}(\%)$ & 352 & $72(20)$ & $280(80)$ \\
Primary indication, $\mathrm{n}(\%)$ & & & \\
$\quad$ Ischaemic heart disease & 152 & $47(31)$ & $105(69)$ \\
$\quad$ Coronary stent & 53 & $26(49)$ & $27(51)$ \\
$\quad$ Coronary bypass surgery & 70 & $18(26)$ & $52(74)$ \\
Cerebrovascular disease & 31 & $7(23)$ & $24(77)$ \\
Atrial fibrillation & 19 & $2(11)$ & $17(89)$ \\
Peripheral vascular disease & 23 & $15(65)$ & $8(35)$ \\
Venous thromboembolic & 10 & $0(0)$ & $10(100)$ \\
Primary prevention & 117 & $1(1)$ & $116(99)$ \\
Surgery, $\mathrm{n}$ (\%) & & & \\
$\quad$ Major vascular & 52 & $36(69)$ & $16(31)$ \\
Major orthopaedic & 80 & $7(9)$ & $73(91)$ \\
General & 48 & $3(6)$ & $45(94)$ \\
Major urological & 66 & $4(6)$ & $62(94)$ \\
Major neurosurgery & 18 & $3(17)$ & $15(83)$ \\
Lower risk & 88 & $19(22)$ & $69(78)$ \\
Revised cardiac risk index, $\mathrm{n}(\%)$ & & & $122(92)$ \\
0 & 133 & $11(8)$ & $108(74)$ \\
1 & 146 & $38(26)$ & $42(74)$ \\
2 & 57 & $15(26)$ & $8(50)$ \\
$\geq 3$ & 16 & $8(50)$ & $9.5 \pm 4.7$ \\
Duration of antiplatelet cessation (days) & & $7(7-10)$ \\
Mean \pm SD & & & \\
Median (25-75th percentile) & & & \\
\hline
\end{tabular}

Values are expressed as mean $\pm \mathrm{SD}$, median (IOR) or $\mathrm{n}(\%)$.

definition, in addition to an elevation above the 99th reference percentile, occurred in $26 \%$ of patients. Only seven patients ( $2 \%$ ) suffered clinically apparent $\mathrm{MI}$ as defined by the current universal definition. ${ }^{17}$ In the initial 86 patients who had both fourth generation $\mathrm{TnT}$ and hs-TnT measured, myocardial necrosis occurred in $6 \%$ and $26 \%$ of patients respectively, resulting in near a fivefold increase in diagnosis using the more sensitive assay. There were no deaths prior to discharge in our cohort. Other major adverse perioperative cardiovascular outcomes occurred infrequently (table 4). Major and minor Thrombolysis in Myocardial Infarction defined bleeding occurred in $7 \%$ and $21 \%$ of patients respectively, with $7 \%$ requiring blood transfusion in the postoperative period.

For patients with Revised Cardiac Risk Index scores of 0, 1, 2 and $\geq 3$, myocardial necrosis occurred in $22 \%, 19 \%, 28 \%$ and

Table 4 Adverse perioperative outcomes

\begin{tabular}{ll}
\hline Variable & Study population \\
\hline Major cardiac event, $\mathrm{n}(\%)$ & \\
Death & $0(0)$ \\
Myocardial infarction (Universal Definition) & $7(2)$ \\
Myocardial necrosis, $\mathrm{n}(\%)$ & \\
$50 \%$ delta & $79(22)$ \\
$30 \%$ delta & $93(26)$ \\
Cerebrovascular event, $\mathrm{n}(\%)$ & $1(<1)$ \\
Venous thromboembolic, $\mathrm{n}(\%)$ & $9(3)$ \\
Atrial fibrillation, $\mathrm{n}(\%)$ & $9(3)$ \\
Bleeding events, $\mathrm{n}(\%)$ & \\
Major TIMI & $23(7)$ \\
Minor TIMI & $74(21)$ \\
Blood transfusion & $26(7)$ \\
\hline
\end{tabular}

Values are expressed as $n(\%)$.

TIMI, Thrombolysis in Myocardial Infarction.
$38 \%$ respectively (figure $2 \mathrm{~A}$ ). Those undergoing major orthopaedic or neurosurgical procedures had significantly higher rates of myocardial necrosis than the other surgical procedures (figure $2 \mathrm{~B}$ ). The majority of major orthopaedic surgery undertaken consisted of large joint replacement, with major neurosurgery predominantly lumbar laminectomy or decompressive procedures.

\section{Predictors of perioperative myocardial necrosis}

Table 5 summarises the univariable and multivariable Cox analysis for predictors of perioperative myocardial necrosis. By multivariable Cox regression analysis, age (OR per year 1.07; $95 \%$ CI 1.03 to $1.10, p<0.001$ ), orthopaedic surgery (OR 2.46; $95 \%$ CI 1.32 to $4.59, \mathrm{p}=0.005)$ and intraoperative hypotension (OR 3.67; 95\% CI 1.69 to 7.94, $\mathrm{p}=0.001$ ) were the only independent predictors of perioperative myocardial necrosis. When the categorical variable intraoperative hypotension was replaced in the multivariable analysis by duration of hypotension, it also predicted perioperative myocardial necrosis (OR 1.10 per 5 -min interval; $95 \%$ CI 1.03 to $1.17, \mathrm{p}=0.004)$. Elevated baseline preoperative hs-TnT and pre-operative cessation of antiplatelet therapy were not predictive of myocardial necrosis.

\section{DISCUSSION}

In our prospective cohort of patients at high cardiovascular risk undergoing elective non-cardiac surgery we found that perioperative myocardial necrosis occurred in $22 \%$ of patients and was clinically undetected in the majority of cases. However, hsTnT defined myocardial necrosis did not result in any hospital mortality. Independent predictors of myocardial necrosis included increasing age, intraoperative hypotension and orthopaedic surgical procedures.

There are several potential mechanisms that contribute to the pathophysiology of perioperative MI, the first being an acute 

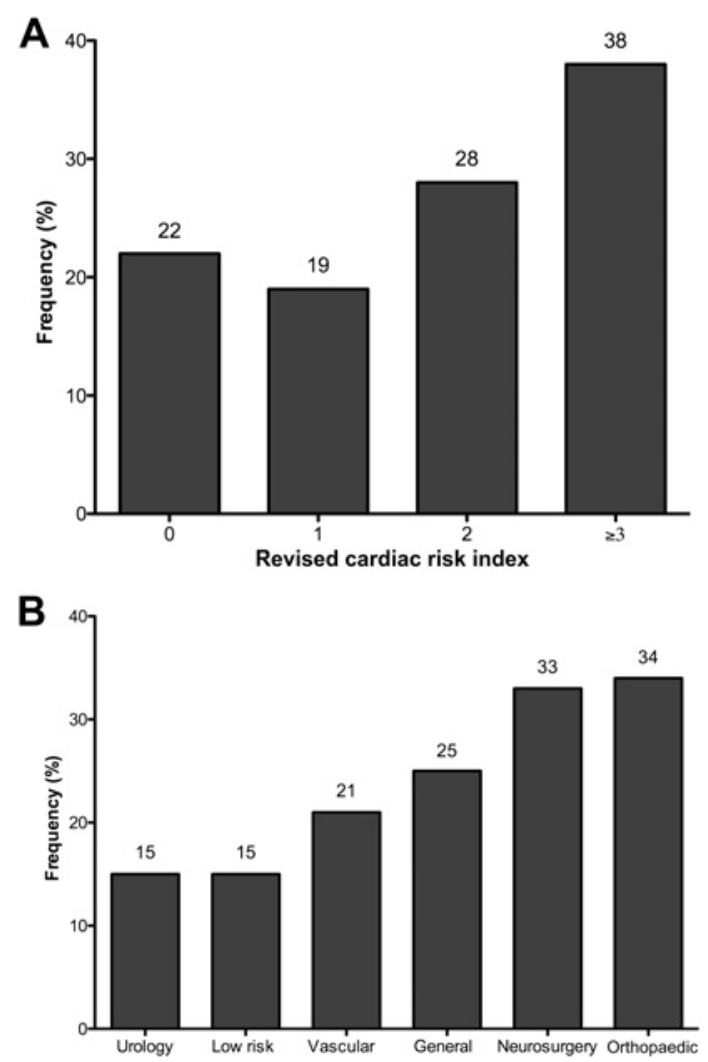

Figure 2 Myocardial necrosis by Revised Cardiac Risk Index (rCRI) and surgery type. (A) Myocardial necrosis by rCRI demonstrating a graded occurrence with increased rCRI score. (B) Myocardial necrosis by surgical type.

coronary syndrome with plaque disruption and resultant thrombus (type 1), the second an imbalance between oxygen supply and demand (type 2), ${ }^{17} 18$ and the third being stent thrombosis, particularly associated with complete interruption of oral antiplatelet therapy. ${ }^{19}$ Reduced oxygen supply for required demand in the perioperative period can be due to multiple factors, including hypotension, hypoxia, anaemia, preexisting coronary stenosis or tachycardia, ${ }^{17} 2021$ and several of these may occur in combination. Intraoperative hypotension was the predominant haemodynamic abnormality in our cohort and increasing duration of hypotension strongly predicted myocardial necrosis. This has previously been identified as an independent predictor of 1-year mortality after non-cardiac surgery. ${ }^{22}$ The relationship of increasing age and myocardial necrosis may reflect an increasing burden of disease, including underlying coronary artery and structural heart disease, in addition to reduced cardiopulmonary reserve. These factors suggest that a 'type 2' mechanism may predominantly underlie perioperative myocardial necrosis and infarction.

Despite the high incidence of hs-TnT-defined myocardial necrosis in our cohort, only $2 \%$ met diagnostic criteria for clinically apparent MI, and there were no deaths in the immediate postoperative period. This suggests in the non-cardiac surgical setting the criteria for MI may need to be broadened to include these adverse events, which are likely to be prognostically significant. Although we included only patients receiving antiplatelet therapy for the primary or secondary prevention of cardiovascular events, the Revised Cardiac Risk Index suggests that our patients were predominantly a lower risk group. The incidence of myocardial necrosis did however occur in a graded manner, rising in line with increasing estimated perioperative risk. This suggests that perioperative hs-TnT elevation may be clinically relevant and that larger studies with longer follow-up might confirm that even minor elevations of hs-TnT are prognostically significant, as has been recently confirmed for less sensitive troponin assays. 81112 Our findings are similar to those reported recently in a similar cohort, where $38 \%$ of patients had postoperative hs-TnT elevations exceeding an $85 \%$ delta value from baseline. ${ }^{23}$

A high incidence of perioperative cardiac events among patients undergoing orthopaedic surgery has been reported previously. Using less sensitive cardiac troponin assays, myocardial necrosis occurs in approximately $6 \%$ of patients undergoing major joint arthroplasty, ${ }^{24}$ and predicts worse longterm outcomes. ${ }^{25}$ Patients undergoing joint replacement surgery tend to be older. However, orthopaedic surgery, predominantly knee and hip replacement surgery in our cohort, remained an independent predictor of perioperative myocardial necrosis even after correction for age and other univariable predictors. Although not investigated in this study, other potential reasons for the high prevalence of hs-TnT elevation after orthopaedic surgery include subclinical fat embolism. This has been reported to occur in up to $67 \%$ of patients following lower-limb joint surgery. ${ }^{26}$ It is plausible that the more significant embolic events may impose haemodynamic cardiac stress through increased pulmonary arterial pressure resulting in the release of troponin.

\section{Preoperative troponin elevation}

Several biomarkers have been shown to strongly predict major cardiovascular events following non-cardiac surgery, including

Table 5 Univariate and multivariate predictors of perioperative myocardial necrosis

\begin{tabular}{|c|c|c|c|c|}
\hline \multirow[b]{2}{*}{ Variable } & \multicolumn{2}{|l|}{ Univariate analysis } & \multicolumn{2}{|l|}{ Multivariate analysis } \\
\hline & OR (95\% Cl) & p Value & OR (95\% Cl) & p Value \\
\hline Age (per year) & 1.06 (1.02 to 1.09$)$ & $<0.001$ & $1.07(1.03$ to 1.10$)$ & $<0.001$ \\
\hline Diabetes (oral therapy) & $1.80(1.00$ to 3.21$)$ & 0.049 & & \\
\hline Prior CABG & $1.66(0.92$ to 2.98$)$ & 0.093 & & \\
\hline ACE-I/ARB therapy & 2.03 (1.11 to 3.72$)$ & 0.021 & & \\
\hline Renal impairment & 1.83 (1.09 to 3.09$)$ & 0.023 & & \\
\hline Haemoglobin concentration & $0.98(0.97$ to 0.99$)$ & 0.026 & & \\
\hline Orthopaedic surgery & $2.16(1.24$ to 3.75$)$ & 0.007 & 2.46 (1.32 to 4.59$)$ & 0.005 \\
\hline Intraoperative hypotension & 3.57 (1.75 to 7.26$)$ & $<0.001$ & 3.67 (1.69 to 7.94$)$ & 0.001 \\
\hline Duration of surgery & 1.01 (1.00 to 1.01$)$ & 0.047 & & \\
\hline Elevated baseline hs-TnT & $1.50(0.89$ to 2.54$)$ & 0.127 & & \\
\hline
\end{tabular}

ACE-I, ACE inhibitor; ARB, angiotensin II receptor blocker; CABG, coronary artery bypass graft; hs-TnT, high-sensitivity troponin T. 
N-terminal pro-B-type natriuretic peptide and C-reactive protein. ${ }^{27}$ However, there are limited data on the prognostic role of elevated preoperative hs-TnT is this setting. In our apparent healthy cohort undergoing elective non-cardiac surgery, we found that $31 \%$ of patients had an hs-TnT level above the 99 th percentile for a healthy population, prior to the planned procedure. This finding is consistent with a recent report in a similar cohort. ${ }^{23}$ It has been demonstrated that circulating levels of hsTnT incrementally predict cardiovascular death and heart failure in both the general population ${ }^{28}$ and those with stable coronary disease, ${ }^{14}$ however this association is not apparent with incident MI. Similarly we demonstrated no relationship between preoperative hs-InT and subsequent perioperative myocardial necrosis. Independent predictors of an elevated preoperative hsTnT in our cohort included age, male gender, diabetes requiring insulin therapy and chronic kidney disease. This is consistent with previous data ${ }^{28}$ and in our study the high proportion of patients with an elevated baseline hs-TnT may reflect the prevalence of these risk factors. Although only a single baseline measurement of hs-TnT was recorded preoperatively, the high prevalence of elevated hs-TnT in an apparent healthy population has important implications for the use of sensitive troponin assays in this setting, and reinforces the need to include a delta value in the definition of perioperative $\mathrm{MI}^{16}$

\section{Cessation of antiplatelet therapy}

Current guidelines recommend against the routine cessation of antiplatelet therapy in patients undergoing elective non-cardiac surgery. ${ }^{29}$ Despite this we found $80 \%$ of all patients had therapy ceased during the perioperative period, occurring a mean of 9 days prior to planned surgery. This occurred despite a high prevalence of established cardiovascular disease, and in half of all patients with previous coronary stents. Although the available data are largely retrospective, they suggest that aspirin withdrawal may precipitate a number of acute cardiovascular events. ${ }^{30-32}$ In addition, approximately $5 \%$ of patients admitted with an acute coronary syndrome have had aspirin therapy ceased in the prior 3 weeks, largely due to scheduled surgery. ${ }^{33}$ Furthermore, antiplatelet therapy interruption more than 5 days preoperatively is independently associated with major adverse cardiac events in patients with coronary stents. ${ }^{19}$ Thus, the routine practice of antiplatelet cessation prior to planned surgery may be detrimental.

Interestingly, we found that antiplatelet cessation was not a predictor of perioperative myocardial necrosis in our cohort. Rather, the independent association of age and intraoperative hypotension with myocardial necrosis suggests that the most common mechanism is likely to be a mismatch between myocardial oxygen supply and demand, rather than thrombus formation on vulnerable plaque, when antiplatelet therapy would be expected to benefit. It might be expected that 'type 1' aetiology of $\mathrm{MI}$ is associated with higher mortality, but our data suggest that these occur less commonly than anticipated.

Although antiplatelet management had no impact on bleeding complications or transfusion requirements, this may be confounded. Patients considered higher risk for bleeding complications may have been more likely to have therapy interrupted compared with those assessed to be at lower risk of bleeding.

\section{LIMITATIONS}

Our observational study describes perioperative practice and outcomes from a single institution including only elective surgery, limiting generalisability. Although hs-TnT-defined myocardial necrosis occurred commonly in our perioperative cohort, it has yet to be correlated with short-term and longerterm adverse events. Routine hs-TnT measurements in our cohort were performed only on the first two postoperative days; it is possible that myocardial necrosis developing after this may have been missed. However, previous studies have demonstrated the majority of events occur in this timeframe. 834 Although we used a $50 \%$ delta value in our cohort, the optimal approach in defining myocardial necrosis, whether a percentage or absolute change, still remains to be fully defined. Finally the study is not adequately powered to assess the impact of antiplatelet management on adverse perioperative cardiac and bleeding events.

\section{CONCLUSION}

In patients with established cardiovascular disease or at risk of the disease who are undergoing major elective non-cardiac surgery, hs-TnT-defined perioperative myocardial injury occurs frequently and is usually clinically undetected. This represents approximately a fivefold increase in diagnosis compared with the previous generation troponin $\mathrm{T}$ assay. Independent predictors of myocardial necrosis include increasing age and intraoperative hypotension, suggesting that supply and demand mismatch may play an important role in cardiac troponin release in this setting. Our findings reinforce the need for thorough preoperative specialist assessment, and highlight the importance of careful intraoperative haemodynamic monitoring.

Contributors All authors contributed equally to the conception, design, data collection and analysis and reporting of data. RFA drafted the manuscript which was reviewed and edited by all other authors. All authors approved the final manuscript.

Funding Dr RFA is supported in part by an unrestricted research grant from the University of Sydney.

Competing interests None.

Ethics approval Ethics approval was approved by Sydney South West Area Health Service Ethics Committee.

Provenance and peer review Not commissioned; internally peer reviewed.

\section{REFERENCES}

1. Weiser TG, Regenbogen SE, Thompson KD, et al. An estimation of the globa volume of surgery: a modeling strategy based on available data. Lancet 2008;372:139-44.

2. Lee $\mathbf{T}$, Marcantonio $\mathrm{E}$, Mangione $\mathrm{C}$, et al. Derivation and prospective validation of a simple index for prediction of cardiac risk of major noncardiac surgery. Circulation 1999;100:1043-9.

3. Poldermans D, Bax J, Kertai M, et al. Statins are associated with a reduced incidence of perioperative mortality in patients undergoing major noncardiac vascular surgery. Circulation 2003;107:1848-51.

4. Devereaux PJ, Yang H, Yusuf S, et al. Effects of extended-release metoprolol succinate in patients undergoing non-cardiac surgery (POISE trial): a randomised controlled trial. Lancet 2008;372:1839-47.

5. Badner N, Knill R, Brown J, et al. Myocardial infarction after noncardiac surgery. Anesthesiology 1998;88:572-8.

6. Ashton C, Peterson N, Wray N, et al. The incidence of perioperative myocardia infarction in men undergoing noncardiac surgery. Ann Intern Med 1993:1187:504-10.

7. Adams J, Sicard G, Allen B, et al. Diagnosis of perioperative myocardial infarction with measurement of cardiac troponin I. N Engl J Med 1994;330:670-4.

8. Devereaux PJ, Xavier D, Pogue J, et al. Characteristics and short-term prognosis of perioperative myocardial infarction in patients undergoing noncardiac surgery. Ann Intern Med 2011;154:523-8.

9. Mackey W, Fleisher L, Haider S, et al. Perioperative myocardial ischemic injury in high-risk vascular surgery patients: incidence and clinical significance in a prospective clinical trial. J Vasc Surg 2006;43:533-8.

10. McFalls E, Ward H, Moritz $\mathrm{T}$, et al. Predictors and outcomes of a perioperative myocardial infarction following elective vascular surgery in patients with documented coronary artery disease: results of the CARP trial. Eur Heart J 2008;29:394-401.

11. Kim L, Martinez E, Faraday N, et al. Cardiac troponin I predicts short-term mortality in vascular surgery patients. Circulation 2002;106:2366-71. 
12. Bursi F, Babuin L, Barbieri A, et al. Vascular surgery patients: perioperative and longterm risk according to the ACC/AHA guidelines, the additive role of post-operative troponin elevation. Eur Heart J 2005;26:2448-56

13. Reichlin T, Hochholzer W, Bassetti S, et al. Early diagnosis of myocardial infarction with sensitive cardiac troponin assays. N Engl J Med 2009;361:858-67.

14. Omland T, de Lemos J, Sabatine M, et al. A sensitive cardiac troponin T assay in stable coronary artery disease. N Engl J Med 2009;361:2538-47.

15. Saenger A, Beyrau R, Braun S, et al. Multicenter analytical evaluation of a highsensitivity troponin T assay. Clin Chim Acta 2011:412:748-54.

16. Chew D, Aroney C, Aylward P, et al. 2011 addendum to the National Heart Foundation of Australia/Cardiac Society of Australia and New Zealand Guidelines for the management of acute coronary syndromes (ACS) 2006. Heart Lung Circ 2011;20:487-502

17. Thygesen $\mathbf{K}$, Alpert J, White $\mathrm{H}$; on behalf of the Joint ESC/ACCF/AHA/WHF Task Force for the Redefinition of Myocardial Infarction. Universal definition of myocardial infarction. Circulation 2007;116:2634-53.

18. Landesberg G, Beattie S, Mosseri M, et al. Perioperative myocardial infarction. Circulation 2009;119:2936-44.

19. Albaladejo P, Marret E, Samama C, et al. Non-cardiac surgery in patients with coronary stents: the RECO study. Heart 2011:97:1566-72.

20. Weiskopf R, Feiner $\mathrm{J}$, Hopf $\mathrm{H}$, et al. Heart rate increases linearly in response to acute isovolemic anemia. Transfusion 2003:43:235-40.

21. Wu W, Schifftner T, Henderson W, et al. Preoperative hematocrit levels and postoperative outcomes in older patients undergoing noncardiac surgery. JAMA 2007:297:2481-8

22. Monk T, Saini V, Weldon B, et al. Anesthestic management and one-year mortality after noncardiac surgery. Anesth Analg 2005;100:4-10.

23. Kavsak $\mathbf{P}$, Walsh $M$, Srinathan $S$, et al. High sensitivity troponin T concentrations in patients undergoing noncardiac surgery: a prospective cohort study. Clin Biochem 2011;44:1021-4.
24. Urban M, Jules-Elysee K, Loughlin C, et al. The one year incidence of postoperative myocardial infarction in an orthopedic population. HSS J 2007:4:76-80

25. Chong C, Lam 0, Ryan J, et al. Impact of troponin I in long-term mortality after emergency orthopaedic surgery in older patients. Intern Med J 2010;40:751-6.

26. Bruce W, Van der Wall H, Peters $M$, et al. Novel imaging strategy for the detection of fat embolism after arthroplasty. ANZ J Surg 2004:74:723-6.

27. Choi JH, Cho D, Song YB, et al. Preoperative NT-proBNP and CRP predict perioperative major cardiovascular events in non-cardiac surgery. Heart 2010;96:56-62.

28. De Lemos J, Drazner M, Omland T, et al. Association of troponin T detected with a highly sensitive assay and cardiac structure and mortality risk in the general population. JAMA 2010;304:2503-12.

29. Poldermans D, Bax J, Boersma E, et al. Guidelines for pre-operative cardiac risk assessment and perioperative cardiac management in non-cardiac surgery. Eur Heart J 2009;30:2769-812.

30. Burger W, Chemnitius J, Kneissl G, et al. Low-dose aspirin for secondary cardiovascular prevention-cardiovascular risks after its perioperative withdrawal versus bleeding risks with its continuation - review and meta-analysis. J Intern Med 2005;257:399-414

31. Biondi-Zoccai G, Lotrionte $\mathrm{M}$, Agostoni $\mathrm{P}$, et al. A systematic review and metaanalysis on the hazards of discontinuing or not adhering to aspirin among 50,279 patients at risk for coronary artery disease. Eur Heart J 2006;27:2667-74.

32. Garcia Rodríguez L, Cea-Soriano L, Martín-Merino E, et al. Discontinuation of low dose aspirin and risk of myocardial infarction: case-control study in UK primary care. BMJ 2011;343:d4094.

33. Collet J, Blanchet B, Tanguy ML, et al. Impact of prior use or recent withdrawal of oral antiplatelet agents on acute coronary syndromes. Circulation 2004:110:2361-7.

34. Le Manach Y, Perel A, Coriat $P$, et al. Early and delayed myocardial infarction after abdominal aortic surgery. Anesthesiology 2005;102:885-91.

Heart online

Visit Heart online for free editor's choice articles, online archive, email alerts, blogs or to submit your paper. Keep informed and up to date by registering for electronic table of contents at heart.bmj.com. 